\title{
EXPLORATORY CHANDRA OBSERVATION OF THE ULTRALUMINOUS QUASAR SDSS J010013.02+280225.8 AT REDSHIFT 6.30
}

\author{
Yanli Ai ${ }^{1}$, Liming Dou ${ }^{2}$, Xiaohui Fan ${ }^{3,4}$, Feige Wang ${ }^{5}$, Xue-Bing Wu ${ }^{4,5}$, and Fuyan Bian ${ }^{6,7}$ \\ ${ }^{1}$ School of Physics and Astronomy, Sun Yat-Sen University, Guangzhou 510275, China; aiyanli@ mail.sysu.edu.cn \\ ${ }^{2}$ Key Laboratory for Research in Galaxies and Cosmology, Astronomy Department, The University of Sciences and Technology of China, \\ Hefei, Anhui 230026, China \\ ${ }^{3}$ Steward Observatory, University of Arizona, 933 North Cherry Avenue, Tucson, AZ 85721, USA \\ ${ }^{4}$ Kavli Institute for Astronomy and Astrophysics, Peking University, Beijing 100871, China \\ ${ }^{5}$ Department of Astronomy, School of Physics, Peking University, Beijing 100871, China \\ ${ }^{6}$ Research School of Astronomy and Astrophysics, Australian National University, Weston Creek, ACT 2611, Australia \\ Received 2016 April 13; revised 2016 May 10; accepted 2016 May 16; published 2016 June 1
}

\begin{abstract}
We report exploratory Chandra observations of the ultraluminous quasar SDSS J010013.02+280225.8 at redshift 6.30. The quasar is clearly detected by Chandra with a possible component of extended emission. The rest-frame $2-10 \mathrm{keV}$ luminosity is $9.0_{-4.5}^{+9.1} \times 10^{45} \mathrm{erg} \mathrm{s}^{-1}$ with an inferred photon index of $\Gamma=3.03_{-0.70}^{+0.78}$. This quasar is X-ray bright, with an inferred X-ray-to-optical flux ratio $\alpha_{\mathrm{ox}}=-1.22_{-0.05}^{+0.07}$, higher than the values found in other quasars of comparable ultraviolet luminosity. The properties inferred from this exploratory observation indicate that this ultraluminous quasar might be growing with super-Eddington accretion and probably viewed with a small inclination angle. Deep X-ray observations will help to probe the plausible extended emission and better constrain the spectral features for this ultraluminous quasar.
\end{abstract}

Key words: quasars: individual (SDSS J010013.02+280225.8)

\section{INTRODUCTION}

A large number of quasars at redshifts of $z \gtrsim 6$ have been discovered, selected with a combination of optical and nearinfrared colors (Mortlock et al. 2011; Venemans et al. 2013; Jiang et al. 2015; Wang et al. 2016). These sources are powerful probes of cosmic reionization (Fan et al. 2006) and offer insights into the process by which massive black holes formed and grew in the early universe. Recently, $\mathrm{Wu}$ et al. (2015) reported the discovery of SDSS J010013.02+280225.8 (hereafter J0100+2802), an ultraluminous quasar with an estimated black hole mass of 12 billion solar masses. With the highest black hole mass and the highest luminosity among all quasars discovered at $z \gtrsim 5$, it sets the tightest constraints on models for massive black hole growth and evolution at early epochs (e.g., Shankar et al. 2009; Volonteri 2010).

$\mathrm{X}$-ray emissions from quasars reveal the conditions in the innermost regions of their accretion-disk corona, and provide information about how the black hole is fed. There are tentative evidences that high-redshift quasars have relatively soft X-ray spectra, indicating that the black holes are accreting near or above the Eddington rate (Grupe et al. 2006; Page et al. 2014), although debates exist (e.g., Just et al. 2007; Moretti et al. 2014). Mildly super-Eddington intermittent accretion may significantly ease the problem of assembling the massive black holes when the universe was young (Madau et al. 2014). Furthermore, high-redshift quasars, such as the known blazars at $z>5$ (Romani et al. 2004; Frey et al. 2010; Sbarrato et al. 2013; Ghisellini et al. 2015), may have powerful jets that provide a means by which the black hole accretes at a rate that breaks the Eddington limit (Ghisellini et al. 2013). The extended lobes produced by jets may be strong in X-ray, rather than in radio, as the dramatic $(1+z)^{4}$ boosts in the energy density of the cosmic microwave background (CMB)

\footnotetext{
${ }^{7}$ Stromlo Fellow.
}

cause inverse Compton scattering to dominate energy loss of relativistic electrons (Fabian et al. 2014).

X-ray observations of $\mathrm{J} 0100+2802$ provide a crucial test for understanding the formation of the most massive black holes in the early universe. In this Letter we report our exploratory Chandra observation of this ultraluminous quasar. Throughout this paper, we adopt the $\Lambda$ CDM cosmology parameters from the Planck Collaboration (2014): $\Omega_{M}=0.315, \Omega_{\Lambda}=0.685$, and $H_{0}=67.3 \mathrm{~km} \mathrm{~s}^{-1}$. We define power-law photon index $\Gamma$ such that $N(E) \propto E^{-\Gamma}$. For the Galactic absorption of SDSS J0100 +2802 , which is included in the model fitting, we use the value of $N_{\mathrm{H}}=5.82 \times 10^{20} \mathrm{~cm}^{-2}$ (Kalberla et al. 2005). All uncertainties are given at $1 \sigma$, unless otherwise specified.

\section{CHANDRA OBSERVATION AND DATA ANALYSIS}

We obtained Chandra observation of SDSS J0100+2802 on 2015 October 16 using the Advanced CCD Imaging Spectrometer (ACIS, Garmire et al. 2003) instrument. The total integration time is $14.8 \mathrm{ks}$. The target was positioned on the ACIS-S3 chip, which was operated in time-exposure mode, with faint telemetry format. The data were processed with standard CIAO version 4.7 (Fruscione et al. 2006), and only events with grades of $0,2,3,4$, and 6 were considered in the analysis. No background flares are present in the observation. We restrict the data to the derived X-ray counts in the observed frame of $0.5-7 \mathrm{keV}$, considering the increasingly uncertain quantum efficiency of ACIS at lower energies and the steeply increasing background at higher energies.

We first carried out source detection with CIAO task WAVDETECT using wavelet transforms (with wavelet scale sizes of $1,2,4,8$, and 16 pixels) and a false-positive probability threshold of $10^{-6}$. The quasar is detected at equatorial coordinates of 01:00:13.038, +28:02:25.75. The X-ray position differs from its optical position by 0.1 arcsec, within the radius (0.6 arcsec) of $90 \%$ uncertainty circle of Chandra ACIS 


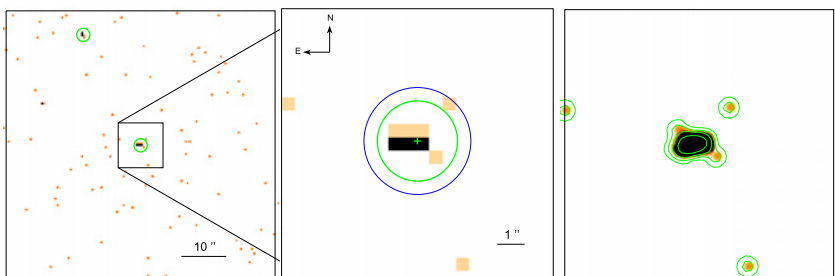

Figure 1. Detection of X-ray emission of J0100+2802. Left: $1^{\prime} \times 1^{\prime}$ Chandra image centered on $\mathrm{J} 0100+2802$ in $0.5-7 \mathrm{keV}$. The circles show the two sources detected by CIAO task WAVDETECT; Middle: central $10^{\prime \prime} \times 10^{\prime \prime}$ of the image. The plus sign shows the optical position given by SDSS. 14 counts are detected in the 3 pixel radius aperture (green), and one more count in the 4 pixel radius aperture (blue). Right: rebinned image in the middle panel to 0.1 ACIS pixel and smoothed with a 0". 492 Gaussion filter. The size of the ACIS CCD pixel is $\sim 0$." 492 .

absolute position. ${ }^{8}$ At a distance of $28^{\prime \prime}$ to the northeast of $\mathrm{J} 0100+2802$, an X-ray source is detected with $7.9 \pm 2.8$ net counts (Figure 1). This source is classified as a galaxy in the SDSS DR12 catalog with a photometric redshift of $0.52 \pm 0.13$ (Alam et al. 2015), therefore it is likely to be a low-luminosity active galactic nucleus in the foreground.

In the image rebinned to 0.1 ACIS pixels and smoothed with a 0.492 Gaussian filter, the spatial distribution for J0100 +2802 appears somewhat elongated (Figure 1). To measure the extension of this source we use Ciao tool "srcextent." While no conclusive result is returned due to the limited source counts of $14-15$. We then manually extracted the photometry of J0100 +2802 and found 14 counts within a 3 pixel $(\sim 1 \prime \prime 5)$ radius aperture, which corresponds to $94.7 \%$ and $89.2 \%$ of the encircled energy fraction in $0.5-2 \mathrm{keV}$ and $2-7 \mathrm{keV}$, respectively. ${ }^{9}$ There is one more count detected at an energy of $1.73 \mathrm{keV}$ within the 4 pixel radius aperture (Figure 1). According to the point-spread function at the source position, the expected count in Poisson statistics is 0.16 in the annulus with radii of 3 and 4 pixels, and 0.78 outside of the aperture with radius of 3 pixels. That is, the chance of this photon belonging to the quasar $\mathrm{J} 0100+2802$ is $14 \%-36 \%$. Thus, for $\mathrm{J} 0100+2802$ we restrict subsequent analysis to the detected photons within the 3 pixel radius aperture. The average background level, estimated from an annulus in the source position with inner and outer radii of 15 and $20 \mathrm{arcsec}$, in an aperture of the source size is 0.06 in $0.5-2 \mathrm{keV}$ and 0.05 counts in $2-7 \mathrm{keV}$.

For J0100+2802, the full-band $(0.5-7 \mathrm{keV}) \mathrm{X}$-ray counts are $13.9_{-3.7}^{+4.8}$, with $11.9_{-3.4}^{+4.6}$ in the soft band $(0.5-2 \mathrm{keV})$ and $2.0_{-1.3}^{+2.6}$ in the hard band $(2-7 \mathrm{keV})$. The errors of the X-ray counts were computed according to Gehrels (1986). The hardness ratio, estimated with the Bayesian method ${ }^{10}$ (Park et al. 2006), is $-0.78_{-0.14}^{+0.07}$, indicating that the X-ray spectrum of $\mathrm{J} 0100+2802$ is soft. The inferred effective photon index from the hardness ratio is indeed large, at $3.2_{-0.4}^{+1.0}$, estimated with Xspec simulation tool "fakeit" and the latest calibration files.

We then perform basic spectral analysis for the quasar. The source spectrum, background spectrum, response matrix files, and auxiliary matrix files are built using the CIAO script SPECEXTRACT. The spectrum is grouped with a minimum of 2 counts per bin. Given that there are only $\sim 14$ net counts, we

\footnotetext{
8 http://cxc.cfa.harvard.edu/cal/ASPECT/celmon/

9 Estimated with the CIAO tool SRC_PSFFRAC.

${ }^{10}$ http://hea-www.harvard.edu/astrostat/behr/
}

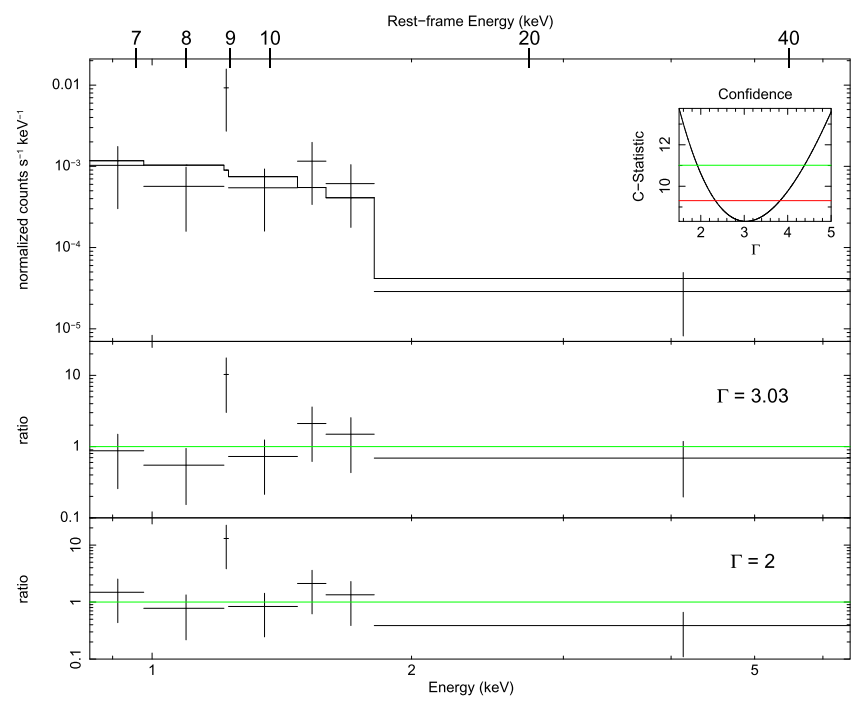

Figure 2. Upper panel: Chandra spectrum of $\mathrm{J} 0100+2802$ and the best-fit power-law model with $\Gamma=3.03\left(N_{\mathrm{H}}\right.$ fixed at Galactic $\left.N_{\mathrm{H}}^{\mathrm{Gal}}\right)$. Inset, confidence curve for the fitted photon index. Middle: data to model ratio of the best-fit model. Significant residual at $\sim 1.2 \mathrm{keV}(8.8 \mathrm{keV}$ at rest-frame) is present in the fitting with different photon indices. Lower: data to model ratio, where a power law with fixed $\Gamma=2.0$ is assumed.

fitted the spectrum using XSPEC (v. 12.9; Arnaud 1996) with a simple power-law model and fixed Galactic absorption. The C-statistic (cstat) was used due to the limited photon counts (Cash 1979). The best-fit photon index is $\Gamma=3.03_{-0.70}^{+0.78}$, with cstat $=8.31$ for 5 degrees of freedom. We also perform simulations taking the exposure time and calibration files, and fit the simulated 1000 spectra. The value of the inferred photon index is consistent with that from the hardness ratio and best fit, with a mean value of 3.13 and a standard deviation of 0.71 .

The best-fit results and confidence curve for the photon index are shown in Figure 2. As a comparison, we also show the fitted spectrum with a fixed photon index of 2.0, the typical value found for quasars. From the data to model ratios we can see that the model with a soft spectral index of 3.03 improves the fitting at the most soft and hard energy bands. The restframe $2-10 \mathrm{keV}$ luminosity implied by the best fit is $9.0_{-4.5}^{+9.1} \times$ $10^{45} \mathrm{erg} \mathrm{s}^{-1}$. There is a significant residual at $\sim 1.2 \mathrm{keV}$ (8.8 keV at rest-frame). There is no known spectral feature at this energy in quasars, and more counts are needed to investigate the nature of this excess.

We label the detected energy and receiving time of each photon in Figure 3. There are 4 photons detected between 1.207 and $1.228 \mathrm{keV}$. This unusual feature causes the significant residual at $\sim 1.2 \mathrm{keV}$ in the spectral fitting (Figure 2). Figure 3 also shows the light curve of $\mathrm{J} 0100+2802$ with a time bin size of $3 \mathrm{ks}$. There are 9 photons detected in the first $3 \mathrm{ks}$ bin, while in the middle $6 \mathrm{ks}$ interval no photon is detected, with a $99 \%$ confidence upper limit of 4.605 (Gehrels 1986). We first test the variation by analyzing the photon arrival time using the Gregory-Loredo algorithm (Gregory \& Loredo 1992) with the CIAO tool GLVARY. The returned variability probability is $P_{\mathrm{GL}}=0.967$, and the variability index is 6 from the test. We then test the chance for a random event of detecting 9 photons in one of the five bins for the total 14 photons with simulation. The chance is 186 in the $10^{5}$ simulated light curves, corresponding to a probability of 


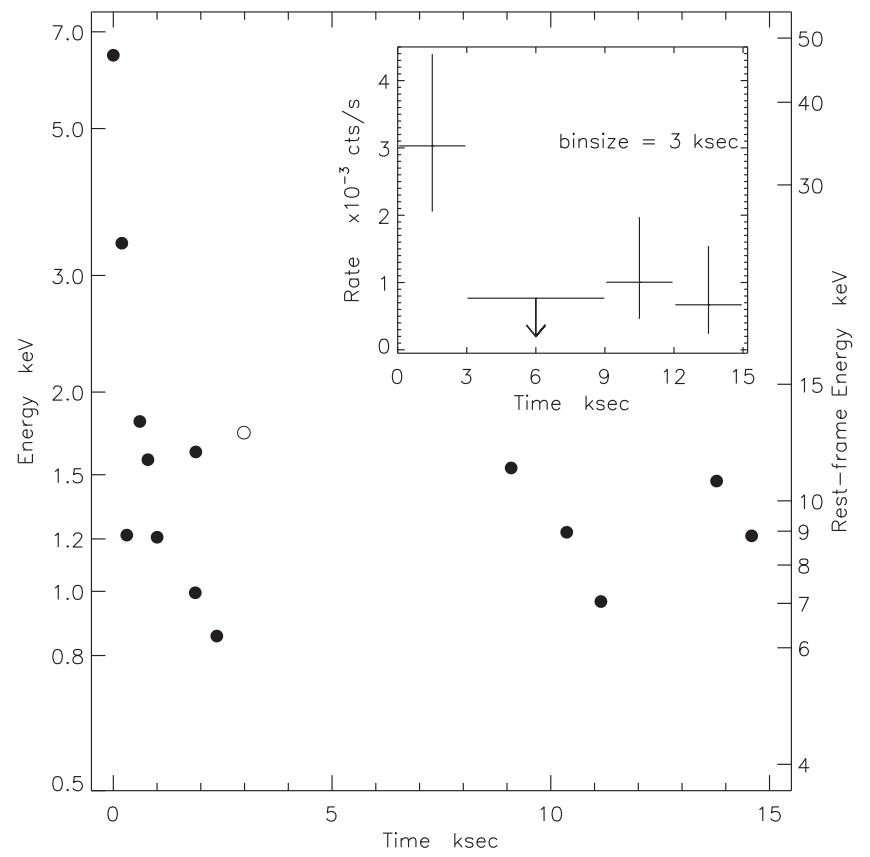

Figure 3. Detected time vs. energy of each photon. The open circle labels the one additional count detected within the 4 pixel radius around the source. Inset, light curve of $\mathrm{J} 0100+2802$ in $0.5-7 \mathrm{keV}$ with a time bin size of $3 \mathrm{ks}$. None of the photons are detected in the middle $6 \mathrm{ks}$. The $99 \%$ confidence upper limit is shown.

$0.019 \%$. Longer exposure observations are needed to investigate this possible variability.

To investigate the broadband properties of $\mathrm{J} 0100+2802$ we calculate the X-ray-to-optical spectral energy distribution parameter, $\alpha_{\text {ox }}$, the slope of a nominal power law between $2500 \AA$ and $2 \mathrm{keV}$ in the rest frame $\left(\alpha_{\mathrm{ox}}=0.384 \times \log \right.$ $\left(f_{2 \mathrm{keV}} / f_{2500 \AA}\right)$, where $f_{2 \mathrm{keV}}$ is the flux density at $2 \mathrm{keV}$ and $f_{2500 \AA}$ is the flux density at $2500 \AA$ ). For $\mathrm{J} 0100+2802$, the rest frame $2500 \AA$ moves to the near-infrared at $18250 \AA$ in the observed frame, falling in the gap between the $\mathrm{H}$ and $\mathrm{K}$ bands, as shown in Figure 3 in Wu et al. (2015). Using the ultravioletoptical continuum slope measured in $\mathrm{Wu}$ et al. (2015), we estimate the flux density at $2500 \AA$ to be $3.04 \pm$ $0.45 \times 10^{-16} \mathrm{erg} \mathrm{s}^{-1} \mathrm{~cm}^{-2} \AA^{-1}$. An energy of $2 \mathrm{keV}$ in the rest frame of $\mathrm{J} 0100+2802$ corresponds to $0.273 \mathrm{keV}$ in the observed frame. This energy is poorly sampled by ACIS. We compute the $0.273 \mathrm{keV}$ flux and the uncertainty from the bestfit power-law model in XSPEC spectral fitting. This yields a value for $\alpha_{\mathrm{ox}}$ of $-1.22_{-0.05}^{+0.07}$, with errors dominated by the uncertainty of the rest-frame $2 \mathrm{keV}$ flux.

\section{DISCUSSION}

The ultraluminous quasar $\mathrm{J} 0100+2802$ is strongly detected in our exploratory Chandra observation with $\sim 14$ counts. We find the X-ray properties of this quasar to be unusual in several aspects.

First, there is a hint of extended emission, and this feature comes from photons in relatively soft energy bands. As first proposed in Fabian et al. (2014), relativistic electrons in powerful jetted objects with a Lorentz factor of $\Gamma \sim 10^{3}$ can upscatter CMB photons into the soft X-ray band. Deeper XMMNewton observations, which have a much higher soft X-ray

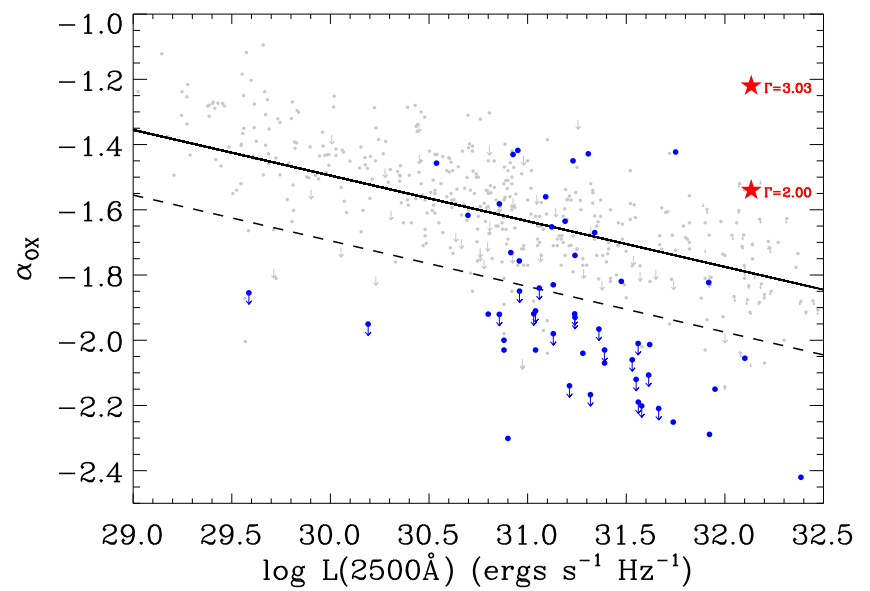

Figure 4. Location of $\mathrm{J} 0100+2802$ (red stars) in the X-ray-to-optical powerlaw slope parameter $\alpha_{\text {ox }}$ vs. $2500 \AA$ monochromatic luminosity. The gray dots are the quasars from the samples of Just et al. (2007), Steffen et al. (2006), and Gibson et al. (2008). The blue dots are the weak-line quasars and PHL 1811 analogs from Luo et al. (2015). The solid line represents the Just et al. (2007) $\alpha_{\mathrm{ox}}-L_{2500 \AA}$ relation, and the dashed line marks the devision between X-ray weak and X-ray normal quasars adopted in Luo et al. (2015). For J0100+2802 the two red stars represent the values of $\alpha_{\mathrm{ox}}$ derived with X-ray photon index of 3.03 and 2.0, respectively.

sensitivity compared to Chandra, would be ideal to investigate the possibility of this extended emission.

Second, the X-ray spectrum of $\mathrm{J} 0100+2802$ is very soft, with an inferred photon index of $3.03_{-0.70}^{+0.78}$, where the mean value of the $\mathrm{X}$-ray photon index found in samples of low- and high-redshift quasars is $1.89<\langle\Gamma\rangle<2.19$ (i.e., Wang et al. 2004; Piconcelli et al. 2005; Just et al. 2007; Young et al. 2009). Although there are still debates about whether the X-ray spectrum of high-redshift quasars significantly differs from that of quasars at lower redshifts (Grupe et al. 2006; Just et al. 2007), we note that the highest redshift quasar ULAS $\mathrm{J} 1120+0641$ may also exhibit a soft X-ray spectrum with a photon index of $2.6_{-0.3}^{+0.4}$ (Page et al. 2014 see Moretti et al. 2014 for a different value of inferred $\Gamma$ ). It has been suggested that the soft X-ray spectrum is an indication that central massive black holes at early epochs are growing at a high accretion rate (Pounds et al. 1995; Leighly 1999).

The inferred $\Gamma$ can be used as an estimator of $L / L_{\text {Edd }}$ by using the linear correlation between $\Gamma$ and $\log \left(L / L_{\text {Edd }}\right)$, where $L$ is the bolometric luminosity and $L_{\mathrm{Edd}}$ is the Eddington luminosity (Shemmer et al. 2008; Risaliti et al. 2009; Jin et al. 2012; Brightman et al. 2013; Yang et al. 2015). Using different relations presented in the literature, we find that the corresponding $L / L_{\mathrm{Edd}}$ for $\mathrm{J} 0100+2802$ has a minimum value of 6 (Risaliti et al. 2009), and can be significantly higher (i.e., Shemmer et al. 2008). If those relations did apply to J0100 +2802 , it could be accreting at significantly above the Eddington rate. Mildly super-Eddington accretion corresponds to a shorter e-folding timescale for black hole mass growth, which can ease the problem of assembling massive black holes out of stellar-mass seeds at early times, even in the case of intermittent accretion (Madau et al. 2014).

Third, J0100+2802 has a relatively high X-ray-to-optical flux ratio compared to those found in other quasars of comparable ultraviolet luminosities (Figure 4). It is well established that the X-ray-to-optical power-law slope parameter $\alpha_{\text {ox }}$ of quasars significantly correlate with the ultraviolet $2500 \AA$ A monochromatic luminosity $\left(L_{2500} \AA\right.$, Steffen et al. 2006; 
Just et al. 2007). According to the $\alpha_{\mathrm{ox}}-L_{2500} \AA$ relation given by Just et al. (2007), a value of $\alpha_{\mathrm{ox}}=-1.79 \pm 0.14$ is expected for $\mathrm{J} 0100+2802$, compared to be the value of $\alpha_{\mathrm{ox}}=-1.22_{-0.05}^{+0.07}$ obtained from the Chandra observation. The observed large $\alpha_{\text {ox }}$ could be due to the inferred steep X-ray photon index, which is still not well constrained based on the current signalto-noise ratio of the data. Even if we adopt a flat X-ray spectral shape with an X-ray photon index of a nominal 2.0 to infer the rest-frame $2 \mathrm{keV}$ flux for $\mathrm{J} 0100+2802$, the derived $\alpha_{\mathrm{ox}}$ is $-1.54_{-0.05}^{+0.07}$, still larger than the average, and is at the upper envelope of the observed $\alpha_{\text {ox }}$ values at comparable ultraviolet luminosities (Figure 4). However, the value of $\alpha_{\mathrm{ox}}$ for J0100 +2802 is still in the range of the ones found in quasars at low to median redshift, supporting that there is no evolution of $\alpha_{\mathrm{ox}}$ with redshift (Brandt et al. 2002; Just et al. 2007; Risaliti \& Lusso 2015).

$\mathrm{J} 0100+2802$ is a weak-line quasar (WLQ) with a rest-frame equivalent width of $\mathrm{Ly} \alpha+\mathrm{Nv} \sim 10 \AA$ (Wu et al. 2015). WLQs are a subclass of radio-quiet quasars that have extremely weak or almost undetectable emission lines (e.g., Fan et al. 1999; Meusinger \& Balafkan 2014 and references therein). The nature of WLQs is still under investigation, although a number of hypotheses have been proposed, such as abnormal broad emission line regions with a significant deficit of line-emitting gas (e.g., Shemmer et al. 2010) or in an early evolutionary stage of formation (e.g., Hryniewicz et al. 2010), a soft ionizing spectral energy distribution due to intrinsic X-ray weakness (e.g., Leighly et al. 2007) or due to small-scale absorption (e.g., Wu et al. 2012; Luo et al. 2015). Significant fractions $(\sim 50 \%)$ of the WLQs are distinctly X-ray weak compared to typical quasars (Shemmer et al. 2009; Wu et al. 2012; Luo et al. 2015). Our results demonstrate that $\mathrm{J} 0100+2802$ is definitely not an X-ray weak WLQ, as shown in Figure 4. If we interpret $\mathrm{J} 0100+2802$ in the scenario proposed by $\mathrm{Wu}$ et al. (2012) and Luo et al. (2015), in which they unified X-ray weak and X-ray normal WLQs by orientation effects with proposed "shielding-gas," this quasar should be viewed at a small inclination angle.

Finally, we detect a hint of X-ray variability for $\mathrm{J} 0100+2802$ within our exposure. For a high-redshift quasar at this luminosity, its X-ray flux is not expected to vary at the timescale of $\sim 10 \mathrm{ks}$ unless beaming is involved. Given that the light crossing time of even the Schwarzschild radius for a 12 billion $M_{\odot}$ black hole is much longer than $10 \mathrm{ks}$, the X-ray flux of $\mathrm{J} 0100+2802$ is not expected to vary in our observations unless strong beaming is involved. Deeper XMM-Newton observations will help to better constrain the spectral features, probe the extended emission, and investigate the variability on both short ( $\sim \mathrm{hr}$ ) and longer (months to year) timescales for this ultraluminous quasar.

Y.-L.A. thanks the support from NSFC grant Nos. 11273060, 11333008, and State Key Development Program for Basic Research of China (Nos. 2013CB834900 and 2015CB857000). F.W. and X.-B.W. thank the support from the NSFC grants No.11373008 and 11533001, the Strategic Priority Research Program "The Emergence of Cosmological Structures" of the Chinese Academy of Sciences, grant No. XDB09000000, and the National Key Basic Research Program of China 2014CB845700. We thank Tinggui Wang, Andy Fabian, Rongfeng Shen, and Yirong Yang and for helpful discussions, and B. Luo for providing the data of Figure 4. This work is based observations made by the Chandra X-Ray Observatory and has made use of software provided by the Chandra X-Ray Center in the application package CIAO. Support for this work was provided by the National Aeronautics and Space Administration through Chandra Award Number GO5-16116B issued by the Chandra X-ray Observatory Center, which is operated by the Smithsonian Astrophysical Observatory for and on behalf of the National Aeronautics Space Administration under contract NAS8-03060.

\section{REFERENCES}

Alam, S., Albareti, F. D., Allende Prieto, C., et al. 2015, ApJS, 219, 12 Arnaud, K. A. 1996, in ASP Conf. Ser. 101, Astronomical Data Analysis Software and Systems V, ed. G. H. Jacoby, \& J. Barnes (San Francisco, CA: ASP), 17

Brandt, W. N., Schneider, S. P., Fan, X. H., et al. 2002, ApJ, 569, 5

Brightman, M., Silverman, J. D., Mainieri, V., et al. 2013, MNRAS, 433, 2485 Cash, W. 1979, ApJ, 228, 939

Fabian, A. C., Walker, S. A., Celotti, A., et al. 2014, MNRAS, 442, 81

Fan, X., Carilli, C. L., \& Keating, B. 2006, ARA\&A, 44, 415

Fan, X., Strauss, M. A., Gunn, J. E., et al. 1999, ApJL, 526, L57

Frey, S., Paragi, Z., Gurvits, L. I., Cseh, D., \& Gabányi, K. É 2010, A\&A, 524, A83

Fruscione, McDowell, J. C., Allen, G. E., et al. 2006, Proc. SPIE, 6270, 62701 Garmire, G. P., Bautz, M. W., Ford, P. G., Nousek, J. A., \& Ricker, G. R. 2003, Proc. SPIE, 4851, 28

Gehrels, N. 1986, ApJ, 303, 336

Ghisellini, G., Haardt, F., Della, R. C., Volonteri, M., \& Sbarrato, T. 2013, MNRAS, 432, 2818

Ghisellini, G., Tagliaferri, G., Sbarrato, T., \& Gehrels, N. 2015, MNRAS, 450,34

Gibson, R. R., Brandt, W. N., \& Schneider, D. P. 2008, ApJ, 685, 773

Gregory, P. C., \& Loredo, T. J. 1992, ApJ, 398, 146

Grupe, D., Mathur, S., Wilkes, B., \& Osmer, P. 2006, ApJ, 131, 55

Hryniewicz, K., Czerny, B., Nikołajuk, M., \& Kuraszkiewicz, J. 2010, MNRAS, 404, 2028

Jiang, L., McGreer, I. D., Fan, X., et al. 2015, AJ, 149, 188

Jin, C., Ward, M., \& Done, C. 2012, MNRAS, 425, 907

Just, D. W., Brandt, W. N., Shemmer, O., et al. 2007, ApJ, 665, 1004

Kalberla, P. M. W., Burton, W. B., Hartmann, D., et al. 2005, A\&A, 440, 775 Leighly, K. M. 1999, ApJS, 125, 317

Leighly, K. M., Halpern, J. P., Jenkins, E. B., \& Casebeer, D. 2007, ApJS, 173,1

Luo, B., Brandt, W. N., Hall, P. B., et al. 2015, ApJ, 805, 122

Lyons, L. 1991, A Practical Guide to Data Analysis for Physical Science Students (Cambridge: Cambridge Univ. Press)

Madau, P., Haardt, F., \& Dotti, M. 2014, ApJ, 784, 38

Meusinger, H., \& Balafkan, N. 2014, A\&A, 568, A114

Moretti, A., Ballo, L., Braito, V., et al. 2014, A\&A, 563, 46

Mortlock, D. J., Warren, S. J., Venemans, B. P., et al. 2011, Natur, 474, 616

Page, M. J., Simpson, C., Mortlock, D. J., et al. 2014, MNRAS, 440, 91

Park, T., Kashyap, V. L., Siemiginowska, A., et al. 2006, ApJ, 652, 610

Piconcelli, E., Jiminez-Bailon, E., Guinazzi, M., et al. 2005, A\&A, 432, 15

Pounds, K. A., Done, C., \& Osborne, J. P. 1995, MNRAS, 277, L5

Risaliti, G., \& Lusso, E. 2015, ApJ, 815, 33

Risaliti, G., Young, M., \& Elvis, M. 2009, ApJL, 700, L6

Romani, R. W., Sowards-Emmerd, D., Greenhill, L., \& Michelson, P. 2004, ApJL, 610, L9

Sbarrato, T., Tagliaferri, G., Ghisellini, G., et al. 2013, ApJ, 777, 147

Shankar, F., Weinberg, D. H., \& Miralda-Escudé, J. 2009, ApJ, 690, 20

Shemmer, O., Brandt, W. N., Anderson, S. F., et al. 2009, ApJ, 696, 580

Shemmer, O., Brandt, W. N., Netzer, H., Maiolino, R., \& Kaspi, S. 2008, ApJ, 682,81

Shemmer, O., Trakhtenbrot, B., Anderson, S. F., et al. 2010, ApJL, 722, L152 Steffen, A. T., Strateva, I., Brandt, W. N., et al. 2006, AJ, 131, 2826

Venemans, B. P., Findlay, J. R., Sutherland, W. J., et al. 2013, ApJ, 779, 24

Volonteri, M. 2010, A\&ARv, 18, 279

Wang, J.-M., Watarai, K.-Y., \& Mineshige, S. 2004, ApJ, 607, 107

Wang, F., Wu, X.-B., Fan, X., et al. 2016, ApJ, 819, 24

Wu, J., Brandt, W. N., Anderson, S. F., et al. 2012, ApJ, 747, 10

Wu, X.-B., Wang, F., Fan, X., et al. 2015, Natur, 518, 512

Yang, Q. X., Xie, F. G., Yuan, F., et al. 2015, MNRAS, 447, 1692

Young, M., Elvis, M., \& Risaliti, G. 2009, ApJS, 183, 17 